\title{
The role of TP53 gain-of-function mutation in multifocal glioblastoma
}

\author{
Lauren R. Olafson ${ }^{1} \cdot$ Manuri Gunawardena $^{1} \cdot$ Sheri Nixdorf ${ }^{1} \cdot$ Kerrie L. McDonald $^{1} \cdot$ Robert W. Rapkins $^{1}{ }^{\circledR}$
}

Received: 20 August 2019 / Accepted: 12 October 2019 / Published online: 30 January 2020

(c) The Author(s) 2020

\begin{abstract}
Purpose The phenotypic and genotypic landscapes in multifocal glioblastoma (MF GBM) cases can vary greatly among lesions. In a MF GBM patient, the rapid development of a secondary lesion was investigated to determine if a unique genetic signature could account for the apparent increased malignancy of this lesion.

Methods The primary (G52) and secondary (G53) tumours were resected to develop patient derived models followed by functional assays and multiplatform molecular profiling.

Results Molecular profiling revealed G52 was wild-type for TP53 while G53 presented with a TP53 missense mutation. Functional studies demonstrated increased proliferation, migration, invasion and colony formation in G53.

Conclusion This data suggests that the TP53 mutation led to gain-of-function phenotypes and resulted in greater overall oncogenic potential of G53.
\end{abstract}

Keywords Glioblastoma $\cdot$ Multifocal $\cdot$ TP53 · Gain-of-function

\section{Introduction}

Glioblastoma (GBM) is the most common and malignant primary brain tumour harbouring few effective treatment options and a poor prognosis. Although often presenting as a solitary tumour (sGBM), GBM can exist as multiple lesions (mGBM), further decreasing the dismal 15 month prognosis to a mere 6-8 months [1-3]. mGBMs are more likely to be deeply disseminated in vital structures, thus preventing gross total resection and resulting in a poorer prognosis compared to sGBM [2]. Incidence of $\mathrm{mGBM}$ in literature ranges from 0.5 to $20 \%$ with a more recent study reporting incidence as high as 35\% [3-5]. Though this apparent increase in incidence likely results from technological advancements in neuroimaging, this prevalence warrants further genetic investigation into $\mathrm{mGBM}$ [2].

Electronic supplementary material The online version of this article (doi:https://doi.org/10.1007/s11060-019-03318-5) contains supplementary material, which is available to authorized users.

Robert W. Rapkins

rapkins@unsw.edu.au

1 Cure Brain Cancer Biomarkers and Translational Research Group, Prince of Wales Clinical School, University of New South Wales, Sydney, NSW 2052, Australia
Dating back to 1963, Batzdorf and Malamud proposed classification of multiple gliomas into multifocal (MF) and multicentric (MC) based on pathological criteria [6]. Maintaining this criterion to present day, MF tumours display a pattern of dissemination whereas MC tumours display no continuity between lesions in the context of time or space, both of which are identified by T2/FLAIR-weighted signals on magnetic resonance imaging (MRI) [6]. With advancements in next generation sequencing (NGS), studies suggest that genetically distinct $\mathrm{mGBMs}$ are rare with most cases likely being MF as opposed to truly MC $[2,7]$. In the present case, two lesions lacked anatomical continuity in imaging diagnostics which, by clinical definition, are considered MC. However, multiplatform molecular examination revealed nearly identical genomic profiles, a case similarly reported by Akimoto and colleagues [7]. These findings suggest that the secondary tumour may have developed either from a subpopulation of the primary tumour or from the evolution of a migrated tumour precursor cell. Considering the genetic similarities between these tumours, this case will be referred to as MF for the purpose of this study. Nevertheless, the definitions of MC or MF offer very little clinical value.

The genomic profiles of GBM have a vast spectrum resulting from significant heterogeneity within the inter- and intra-tumoural landscape. Additionally, the diffusely infiltrative nature of the disease has had significant implications on 
diagnostic and therapeutic advancements. Despite improved surgical techniques and anticancer drugs, current treatment options for sGBM are limited to resection followed by concurrent radiotherapy and chemotherapy [1]. Treatment options for mGBM are inevitably more complicated and remain controversial [5]. Where the 5-year survival rate has improved for other malignancies including breast and prostate cancer, no improvements for GBM have been recorded in the past 20 years. Thus, an understanding of the aggressive biology and tumour evolution of this formidable disease is urgently needed.

Coined the "guardian of the genome", the tumour suppressor gene TP53 has garnered significant research attention. It is the most commonly mutated gene found in all human cancers and consequently, the most extensively studied gene of all time. Alterations in TP53 and its encoding protein, p53, have been found in approximately half of all human cancers with the majority of mutant proteins resulting from missense mutations [8-10]. Previous studies have demonstrated that these alterations not only result in a loss of wild-type (wt) function, but can acquire gain of function (GOF) phenotypes rendering the cancer more aggressive [11]. These GOF phenotypes may potentiate aggressive tumour progression through increased cell migration, proliferation, invasion and metastasis providing the mutant isoforms with greater oncogenic potential than p53 wt loss [11-14].

Most of the investigative findings on p53 GOF phenotypes have been conducted through in vitro and in vivo studies, but little has been reported within a more relevant clinical setting. Herein, we present a 55-year-old male patient with MF GBM presenting in the left thalamic $(4.7 \mathrm{~cm})$ and left temporal $(5.4 \mathrm{~cm})$ regions with the latter developing in less than two weeks after discovery of the thalamic lesion. Following resection, fresh primary and secondary tumour samples were collected and processed for patient derived model development. Multi-platform molecular profiling was conducted on both samples with additional standard of care diagnostics. Molecular profiling revealed a TP53 missense mutation with subsequent functional studies identifying p53 GOF phenotypes in the secondary tumour. This case report emphasises the complex GBM landscape and thus, the potential contribution of genetic analysis and interpretation in formulating personalised treatment plans.

\section{Materials and methods}

\section{Patient-derived cell line establishment and tissue culture}

A 55-year-old male patient presented with left-sided headache, dysphasia, significant right proximal arm weakness and peri-orbital pain. Imaging revealed an enlarged left thalamic lesion $4.7 \mathrm{~cm}$ in diameter. The patient was scheduled for surgical resection within two weeks at The Prince of Wales Private Hospital (Randwick, Australia). The Human Research Ethics Committee, South Eastern Sydney Local Health District-Northern Sector approved the collection and use of fresh human GBM tissue for this project (HREC No: 2008-094). Pathology confirmed extensive palisading necrosis and vascular proliferation consistent with GBM, World Health Organisation grade IV. Preoperative imaging on the day of surgery revealed an additional lesion in the left temporal region measuring $5.4 \mathrm{~cm}$ in diameter (Fig. 1). Samples $2-3 \mathrm{~cm}$ in diameter

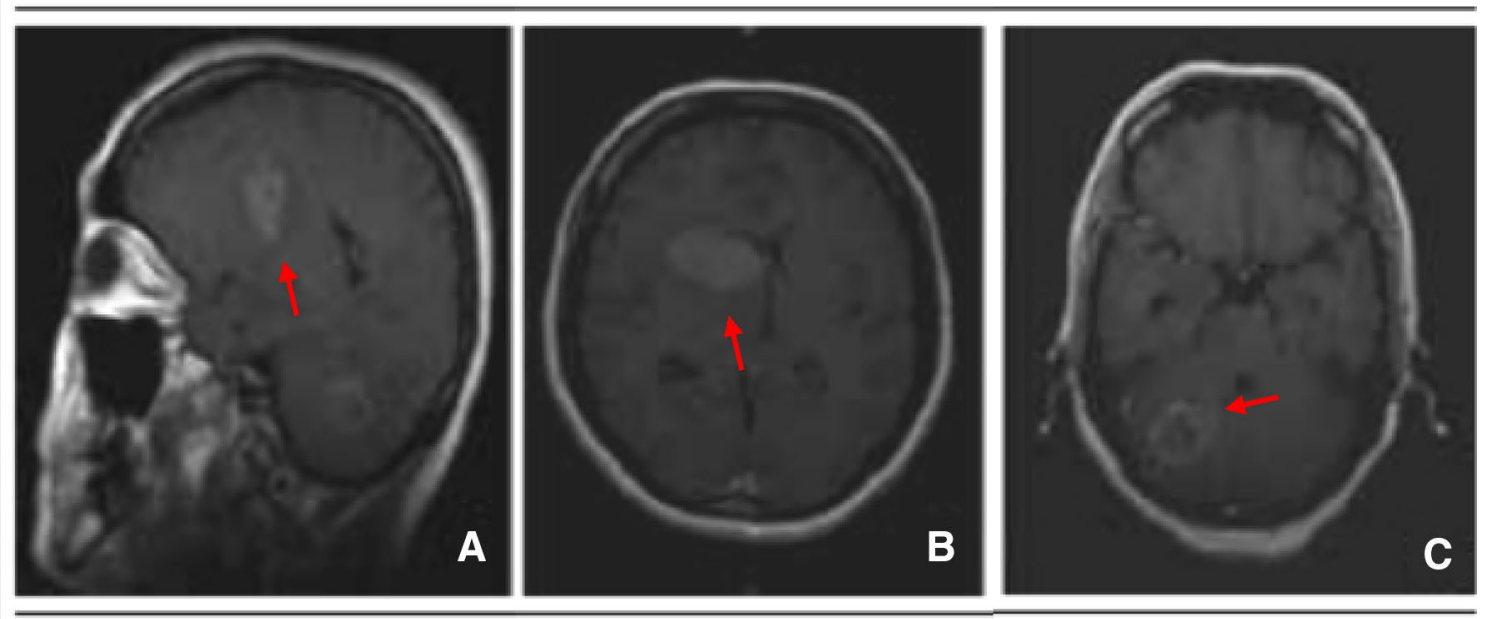

Fig. 1 Pre-operative MRI scans exhibiting two expansive bulky lesions arising in the left thalamus (a and $\mathbf{b}$ ) and cerebella hemisphere $\mathbf{c}$ with an irregular ring contrast enhancement 
from both lesions were collected and transported on ice for processing within 30-60 min of resection. Tissue fragments were washed with phosphate buffered saline (PBS) following the removal of necrotic and vascular regions under a dissecting microscope. A cell pellet was established and resuspended into $10 \mathrm{ml}$ of serum-free media supplemented with $50 \mu 1$ of epidermal growth factor and fibroblast growth factor before being plated onto a T75 flask pre-coated with Corning ${ }^{\circledR}$ Matrigel ${ }^{\circledR}$. Low passage, patient-derived primary cell lines (PDCLs) were established as G52 (left thalamic) and G53 (left temporal) and maintained for subsequent analyses.

\section{Multiplatform molecular profiling of tumour}

Formalin-fixed paraffin-embedded (FFPE) tumour tissue samples of G52 and G53 were sent for multiplatform profiling to Caris Life Sciences, Phoenix, AZ (Molecular Intelligence Service ${ }^{\mathrm{TM}}$ ). Commercially available antibodies and detection kits were used for immunohistochemical (IHC) analysis of PD-1 (NAT1 antibody, Cell Marque), PD-L1 (SP142, Spring Bioscience) and EGFR (Invitrogen) expression. PD-1 expression on the plasma membrane of tumour infiltrating lymphocytes was examined and the density recorded. PD-L1 membrane expression on $>5 \%$ of tumour cells was measured as positive [15]. EGFR expression was assessed using a $\mathrm{H}$-score grading system between 0 and 300. To ensure validity of results, all IHC assays consisted of positive and negative controls. EGFR gene alterations were evaluated for copy number changes using in situ hybridisation assays: chromogenic (CISH [Ventana, Tucson, AZ]) and fluorescent (FISH [Abbott Molecular/ Vysis]). Amplification of EGFR was recognised if $>10 \%$ of analysed cells contained $>15 \%$ EGFR gene copies per well or if the EGFR/CEP7 ratio was $>2$ [16]. FISH was also performed to detect $1 \mathrm{p} 19 \mathrm{q}$ co-deletion and both FISH/CISH were used to detect cMET gene amplification. EGFRvIII mutational analysis was performed on RNA extracted from tumour tissue samples using fragment analysis sequencing and multiplex ligation-dependent probe amplification. NGS analysis was performed on tumour DNA using the Illumina MiSeq platform. Specific regions of 594 genes were amplified (Supplementary Tables 1 and 2) using the Illumina TruSeq AmpliconCancer Hotspot Panel. A > 99\% confidence was detected on all variants based on amplicon coverage and the frequency of mutations present. Sample regions sequenced achieved an average depth of coverage of $>1500 x$. Sanger sequencing was performed on selected regions of IDH2, KRAS, EGFR, c-KIT, BRAF, PIK3Ca and NRAS using
PCR primers designed to amplify target sequences. Methylation testing of $M G M T$ was achieved through pyrosequencing analysis of $\mathrm{CpG}$ sites. Samples were considered equivocal between $\geq 7 \%$ and $<9 \%$ methylation.

\section{Amplification and sanger sequencing to validate TP53 mutations}

Mutations in the TP53 tumour suppressor gene were validated using isolated genomic DNA from G52 and G53 cell lines by PCR amplification followed by Sanger DNA sequencing. The most informative coding regions and intron/ exon junctions of TP53 (exons 5 through to 8) were amplified. The most frequent sites for mutation were represented by three hot spots at amino acids 175 (exon 5), 248 (exon 7) and 273 (exon 8). The specifically designed primer pairs used were 5'-TGT TCA CTT GTG CCC TGA CT-3' (forward) and 5'-TAA CCC CTC CTC CCA GAG A-3' (reverse) covering a 0.550-kilobase region of exons 5 and 6, pairs 5'-AGG CGC ACT GGC CTC ATC TT-3' (forward) and 5'-TGT GCA GGG TGG CAA GTG GC-3' (reverse) spanning a 0.283 -kilobase region of exon 7 and pairs $5^{\prime}$ - TTG GGA GTA GAT GGA GCC T-3' (forward) and 5'-AGT GTT AGA CTG GAA ACT TT-3' (reverse) covering a 0.445-kilobase region of exon 8. Real-time PCR amplification and detection was performed following the manufacturer's protocol using KAPA-Taq Polymerase Kit (Kapa Biosystems): initial denaturation $\left(2 \mathrm{~min}\right.$ at $\left.95^{\circ} \mathrm{C}\right), 35$ cycles of denaturation $\left(95^{\circ} \mathrm{C}\right)$, annealing $\left(61^{\circ} \mathrm{C}\right)$ and extension $\left(72{ }^{\circ} \mathrm{C}\right)$ with a final extension cycle $\left(1 \mathrm{~min}\right.$ at $\left.72^{\circ} \mathrm{C}\right)$. The success of the PCR was verified by running a $5 \mu \mathrm{L}$ aliquot of the PCR product on a $1.6 \%$ agarose gel. Sanger sequencing was performed on the PCR products following the manufacturer's protocol using BigDye ${ }^{\circledR}$ Terminator Cycle Sequencing Kit v3.1 (Applied Biosystems), 45 cycles were performed using an annealing temperature of $56^{\circ} \mathrm{C}$. Mutational data was collected on ABI Prism 3730xl Genetic Analyzer (Applied Biosystems).

\section{Immunohistochemistry}

IHC staining of FFPE sections for Ki-67, an astrocytic proliferative marker, was performed. The sections were deparaffinised with xylene then rehydrated with decreasing concentrations of alcohol. The slides were then washed for $5 \mathrm{~min}$ in PBS. Heat induced antigen retrieval was performed by immersing the sections in $10 \mathrm{ml}$ of citrate buffer pH 6 (Dako, Glostrup, Denmark) and microwaving on high $2 \times 5 \mathrm{~min}$ each. Slides were removed and allowed to cool in solution for $20 \mathrm{~min}$ then washed with PBS for $5 \mathrm{~min}$. Endogenous peroxidase activity was exhausted by treating 
slides with 3\% hydrogen peroxide solution. Tissues were then covered with two drops of endogenous biotin blocking agents, $0.1 \%$ Avidin and Biotin. After washing with PBS, the sections were blocked for $1 \mathrm{~h}$ using $2 \%$ Bovine Serum Albumin in PBS. Each slide was then incubated with the Ki-67 monoclonal primary antibody (clone MIB-1; DAKO, Glostrup Denmark) at room temperature for $1 \mathrm{~h}$. Sections were then washed three times with PBS. A brown colour was developed by applying diaminobenzidine DAB chromogen (DAKO K3456) substrate for 5-10 min followed by haematoxylin counterstaining. As a result, Ki-67 positive nuclei appear brown. Ki-67 expression is defined as a percentage score of the total number of tumour cells with positive nuclear staining per 1000 cells. Viable infiltrating areas on the cancer tissue were selected using $\mathrm{a} \times 10$ objective magnification for analysis. Vascular components, haematogenous tumour cells and non-specific cytoplasm staining were excluded from the analysis. To determine the ratio of positive cells, $\times 40$ magnification was used to count cells showing positive staining. From each chosen area, 1000 cells were counted in consecutive fields. Cells were considered $\mathrm{Ki} 67+$ if there was clearly detectable dark brown colouration of the nucleus.

\section{Cell proliferation assay}

Cell Proliferation was assessed using the xCELLigence Real-Time Cell Analysis (RTCA) system (Roche, Switzerland) on Matrigel coated CIM-16 xCELLigence plates. The E-96 plate consists of incorporated gold cell sensor arrays which allow for the monitoring of cells inside each well. Electronic impedance of the sensors was measured through the detection of cells adhering to the electrodes. Cell attachment acts as insulation altering the electrode/solution interfaces, thereby increasing impedance. The E-96 plate was connected to the RTCA system and background impedance was measured. G52 and G53 cells were seeded onto the plate at an optimised density of $8 \times 10^{3}$ cells per well. The plate was connected to the RTCA system and incubated at $37{ }^{\circ} \mathrm{C}$. Cell adhesion, growth and proliferation were measured every $15 \mathrm{~min}$ for $48 \mathrm{~h}$ via the incorporated sensor electrode rays. Four replicates of the cell concentration were performed in each test. Electrical impedance was measured by the xCELLigence RTCA software as an arbitrary parameter labelled Cell Index (CI).

\section{Cell invasion and migration assay}

Cell migration and invasion assays were conducted using RTCA as previously described. The experiment was performed on CIM-16 plates consisting of an upper and lower chamber separated by an artificial microporous membrane. Migration of cells was detected by microelectrodes attached to the underside of the membrane. Background signals generated by cell-free media were measured. For migration experiments, an optimised density of $4 \times 10^{4}$ cells per well were seeded onto the upper chamber of the CIM-16 plates in serum free medium. The lower chambers were filled with 5\% FCS, used as a chemoattractant. The invasion experiments followed an identical protocol with the additional application of a Matrigel layer to the upper side of the membrane. The chambers were incubated at $37{ }^{\circ} \mathrm{C}$ for a minimum of 4 $\mathrm{h}$ prior to seeding cells. Cells were seeded at an optimised density of $2 \times 10^{4}$ cells per well. CI was measured over $35 \mathrm{~h}$ using RTCA software. Original datasets generated by xCELLigence were exported to MS Excel and reconstructed with data points corresponding to selected timepoints.

\section{Colony formation assay}

The ability for a single cell to develop into a colony can be measured in vitro by colony formation assays. There must be at least 50 cells or more in a grouping to constitute a colony. Colony formation assays were performed on both G52 and G53 cell lines. Cells were harvested from culture at $80 \%$ confluency and trypsinised into single cell suspensions. A cell count was performed. Cells were seeded onto individual 6 well plates at concentrations of 50, 100, 200, 300, 400 and 600 cells $/ \mathrm{ml}$. Plates were incubated at $37^{\circ} \mathrm{C}$ and cell attachment was analysed under a microscope after $24 \mathrm{~h}$. Cells were left in a $37{ }^{\circ} \mathrm{C}$ incubator for 14 days. The plates were then stained with a $6 \%$ glutaraldehyde and $0.5 \%$ crystal violet mixture for $30 \mathrm{~min}$ and rinsed with water. A stereomicroscope and colony counting pen were used to count colonies.

\section{Results}

\section{Biomarkers and mutations revealed by molecular profiling}

A total of 27 biomarkers were tested in the samples using multiplatform molecular profiling involving in situ hybridisation, IHC, Sanger sequencing, fragment analysis and NGS with a further 594 genes amplified using the Illumina TruSeq Amplicon-Cancer Hotspot Panel. No MGMT promoter methylation was identified in either tumour and biomarkers that tested positive were identical in both (Table 1). Mutational analysis revealed 4 of the 594 genes analysed were mutated in both tumours. Further NGS analysis revealed that G52 somatic single nucleotide variations and indel mutations shared $92 \%$ similarity with G53. The only variance noted 
Table 1 Positive biomarkers and mutated genes analysed by pyrosequencing, in situ hybridisation, IHC and NGS for G52 and G53 tumour samples

\begin{tabular}{lll}
\hline Pyrosequencing & G52 Tumour & G53 Tumour \\
\hline $\begin{array}{l}\text { Pyro SEQ-MGMT } \\
\text { In situ hybridisation }\end{array}$ & Unmethylated & Unmethylated \\
EGFR & Positive & Positive \\
IHC & & \\
EGFR & Positive & Positive \\
PTEN & Positive & Positive \\
TOPIIA & Positive & Positive \\
PGP & Positive & Positive \\
Next generation sequencing & & \\
$P T E N$ & Mutated & Mutated \\
EGFR & Mutated & Mutated \\
$M A P 3 K I$ & Mutated & Mutated \\
$N T R K I$ & Mutated & Mutated \\
TP53 & Wild-type & Mutated \\
\hline
\end{tabular}

between the two tumours was observed in TP53, presenting as wt in G52 and mutated in G53. Altogether, the biomarker and mutational data revealed similar molecular profiles of the MF lesions, differing only in a TP53 mutation.

\section{Mutational validation of TP53 in multifocal glioblastoma lesions}

Genotyping of the 'hotspot' codons of TP53 (175, 248 and 273) in both tumours confirmed missense mutation c.818G > A (p.R273H) in exon 8 of G53 (Fig. 2b). Homozygotic substitution of coding DNA sequence (C:G > T:A) has been previously annotated as a single nucleotide polymorphism (SNP) in the dbSNP database (rs28934576).

\section{Mutant p53 gene promotes tumour proliferation, migration and invasion in human GBM cells}

To investigate the potential functional role of the p53 mutation in G53, cell proliferation, migration and invasion were monitored in real-time using xCELLigence technology for each tumour cell line. The rate of cell proliferation was increased in the G53 cells (p53 mutant) when compared with G52 cells (p53 wt) for the same time period (Fig. 3a). At the single time-point of $24 \mathrm{~h}$, the G53 cell index (CI) count was $9 \times$ greater than G52 (Fig. 3b). The migratory properties of the tumour cells were investigated using uncoated CIM-16 xCELLigence plates. The rate of migration through the uncoated membrane was $3 \times$ greater in G53 cells. The migratory rate of G53 continually increased after the $13 \mathrm{~h}$ timepoint compared to

A

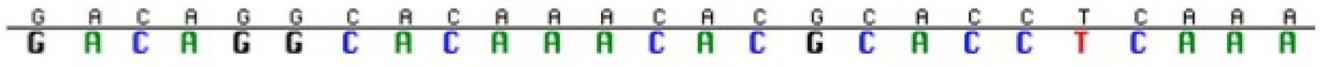

Wildtype

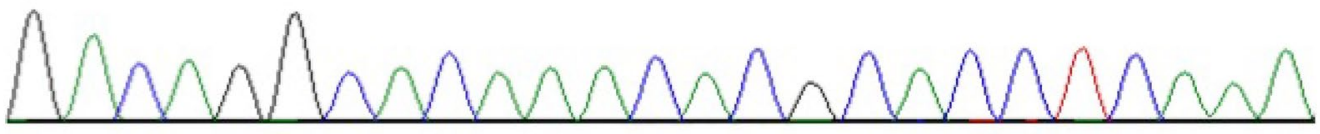

B

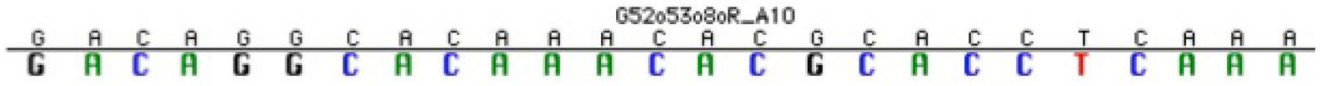

G52

Exon 8

Reverse

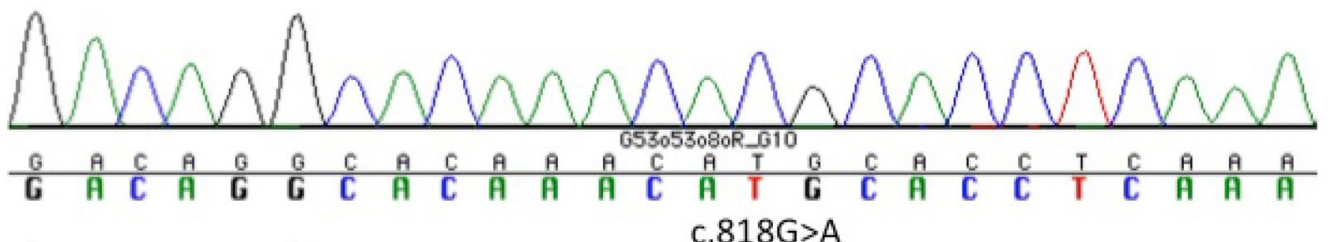

G53

Exon 8

Reverse

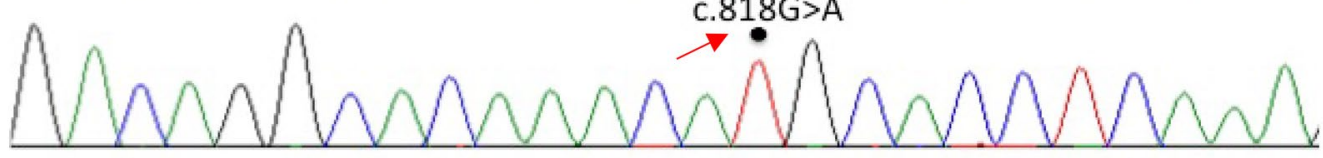

Fig. 2 Nucleotide sequence analysis of TP53 exon 8 in G52 and G53 tumour samples. a Wt nucleotide sequence at codon 273. b

G52 and G53 tumour nucleotide sequence at codon 273. Dot indi- cates the position of the $\mathrm{C}$ to $\mathrm{T}$ nucleotide substitution and c.818G.A (p.R273H) mutation of the TP53 gene in the G53 tumour 
A

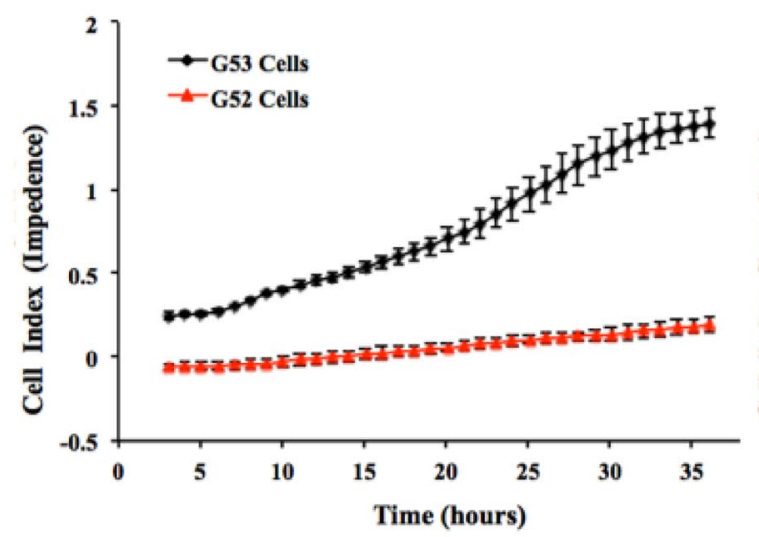

C

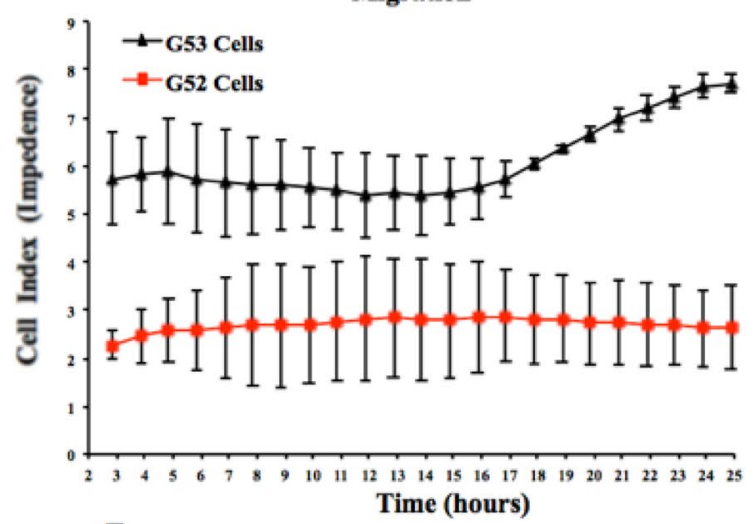

E

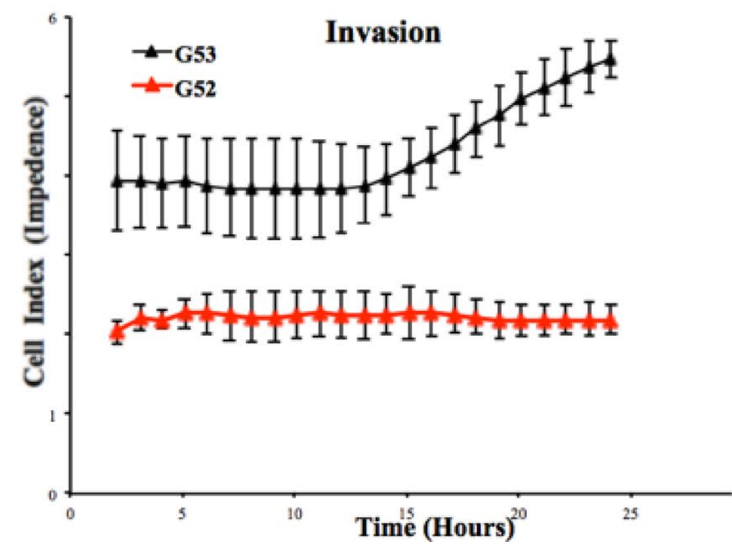

Fig. 3 p53 mutation promotes cell proliferation, migration and invasion in multifocal primary glioblastoma cell lines in vitro. a Realtime xCelligence analysis of proliferation (represented by cell index) of G52 (red) and G53 (black). b $24 \mathrm{~h}$ timepoint analysis of proliferation (represented by cell index) levels between G53 and G52. Error bars represent standard deviations. $p<0.1 ; * p<0.02$. c Real-time xCelligence analysis of migration (represented by cell index) of G53 and G52. d 24 h timepoint analysis of migration (represented by cell

the G52 cells that maintained a lower and constant rate of migration throughout the same period (Fig. 3c). At the single timepoint of $24 \mathrm{~h}$, the G53 CI count for migration
B

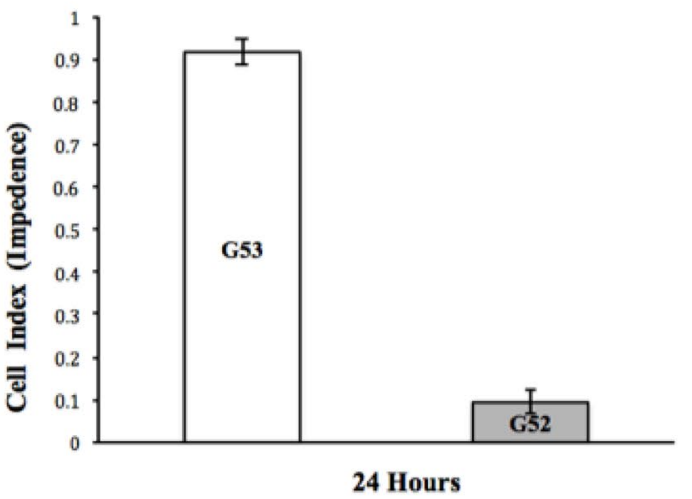

D
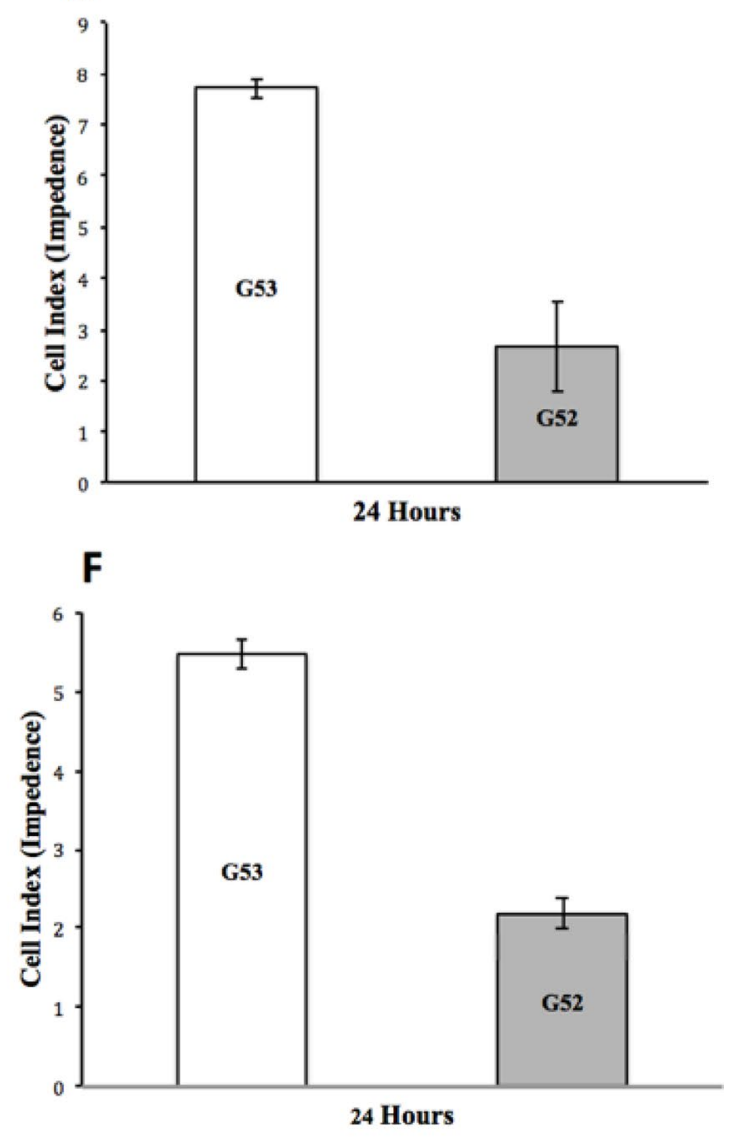

index) levels between G53 (left frontal lesion) and G52 (left thalamic lesion). Error bars represent standard deviations. $p<0.1 ; p<0.8$. e Real-time xCelligence analysis of invasion (represented by cell index) of G53 (black) and G52 (red). f 24 h timepoint analysis of invasion (represented by cell index) levels between G53 (left frontal lesion) and G52 (left thalamic lesion). Error bars represent standard deviations. $p<0.2 ; p<0.1$

was greater than G52 (Fig. 3d). The invasive properties of the cell lines were investigated using CIM-16 xCelligence plates coated with Matrigel ${ }^{\circledR}$. The rate of cell invasion 
through Matrigel was increased by $2.5 \mathrm{x}$ in G53 cells compared with G52 (Fig. 3e). The invasiveness of G53 cells increased sharply after $13 \mathrm{~h}$. At the single timepoint of $24 \mathrm{~h}$, the G53 cell index count for invasion was greater than G52 (Fig. 3f). As expected, these results indicate that the p53 mutant cell line represents a more aggressive tumour that promotes proliferation, migration and invasion of GBM cells in vitro.

\section{In vitro expression of wild-type and mutant p53: effect on colony formation}

A colony formation assay was used to determine the potential and differences of both the wt and mutant p53 tumours (Fig. 4). There are clear differences between the two tumour populations with G53 forming significantly more colonies across all cell densities. Figure $4 \mathrm{a}$ demonstrates consistent increases in G53 colony formation (crystal violet stain) as cell density increases while G52 colonies cannot be visualised in any of the plates. These differences are quantified in Fig. 4b. These results suggest G52 maintained p53 wt tumour suppressor function whereas G53 p53 mutant supported cellular growth.

\section{Ki-67 expression in G52 and G53 tumour tissue}

The labelling index (LI) for Ki-67 was calculated as the percentage of positive cells per 1000 cells (Fig. 5). The percent of Ki-67 significantly increased in G53 ( $p<0.05$; Fig. $5 b$ ), suggesting that the p53 mutation was associated with the $\mathrm{Ki}-67 \mathrm{LI}$ indicating positive proliferation.

\section{Discussion}

GBM is a molecularly complex disease resulting in extensive diagnostic and therapeutic challenges. Consequently, the standard of care has remained unchanged since the Stupp protocol was introduced in 2005, consisting of maximal safe surgical resection followed by radiotherapy plus concomitant and adjuvant temozolomide [1]. Treatment is further complicated when mGBM cases arise where the prognosis declines to 6-8 months and no standard of care exists. MC tumours are defined as lesions in different compartments of the brain with no gross or microscopic anatomical linkages. MF tumours may disseminate through established CNS routes including cerebrospinal fluid, white matter or through local invasion. However, GBMs are highly invasive and visible anatomical discontinuity does not necessarily insinuate genomic isolation between tumours. Microscopic pathways may exist that cannot be detected even through the most advanced imaging technology. Although there was no apparent FLAIR pattern of dissemination in this patient, similar genomic profiles suggest that the tumours shared a common origin, findings consistent with previous case reports $[7,17]$. Moreover, GBMs are known to be a clonal disease originating from normal neural stem cells and MF cases are assumed to follow this trend through migration and mutation events [18-20].

A more intensively studied topic relative to $\mathrm{mGBM}$ is the TP53 tumour suppressor gene, which confers pivotal protective functions against cancer through the regulation of cell proliferation and death. However, it is also the most commonly mutated gene found in nearly half of all human cancers. In glioma patients, TP53 mutations have been found to have a direct negative impact on overall survival [21]. Compromises to gene functionality can occur either through attenuation via missense mutations or by allelic deletion with the former rendering a more aggressive and metastatic cancer. The majority of p53 mutants (75\%) are a result of missense mutations found within the DNA-binding domain [22]. Here, they may either alter the structure of the domain (conformational mutant) or hinder contact between p53 mutants and DNA (contact mutant). Of these $75 \%$, six frequently occur within "hotspot" codons, namely R175, G245, R248, R249, R273 and R282, which are responsible for $30 \%$ of the missense mutations [22]. These mutations result in an accumulation of highly stabilised mutant proteins within the nucleus. $\mathrm{R} 273 \mathrm{H}$ ( $\mathrm{R} 270 \mathrm{H}$ in mice) is one of the more common and least stable of the hotspot mutations and was found to be unique in G53 [23, 24]. It is not thought to undergo conformational changes, but rather maintains the wt structure while affecting surface proteins essential for DNA binding (contact mutant) [22-24].

We anticipated distinct mutational profiles that could explain the sudden appearance and rapid development of G53. However, multiplatform profiling revealed that the positive biomarkers as well as the mutated driver genes were similar in both tumours with the only significant difference being a p53 R273H mutation in G53. Although the exact pathogenic mechanisms of MF tumours remain unknown, we hypothesise that tumour cells migrated from the left thalamus to the left temporal region despite being well-separated lesions displaying no apparent pattern of dissemination. A previous study has suggested that mGBMs are of monoclonal origin and identified potential founder events involving loss of one copy of chromosome 10 with PTEN, EGFR and TERT promoter mutations followed by further alterations, including TP53 mutations [25]. The present study identified $P T E N$ and EGFR mutations in both tumours with loss of one copy of chromosome 10 and a TP53 mutation in G53. Due to the heterogeneic nature of GBM, we propose the possible mechanisms for which TP53 mutated: (i) in a clonal event during a later stage of G52 tumour development before branching off to form G53 or (ii) parallel genetic evolution 
A
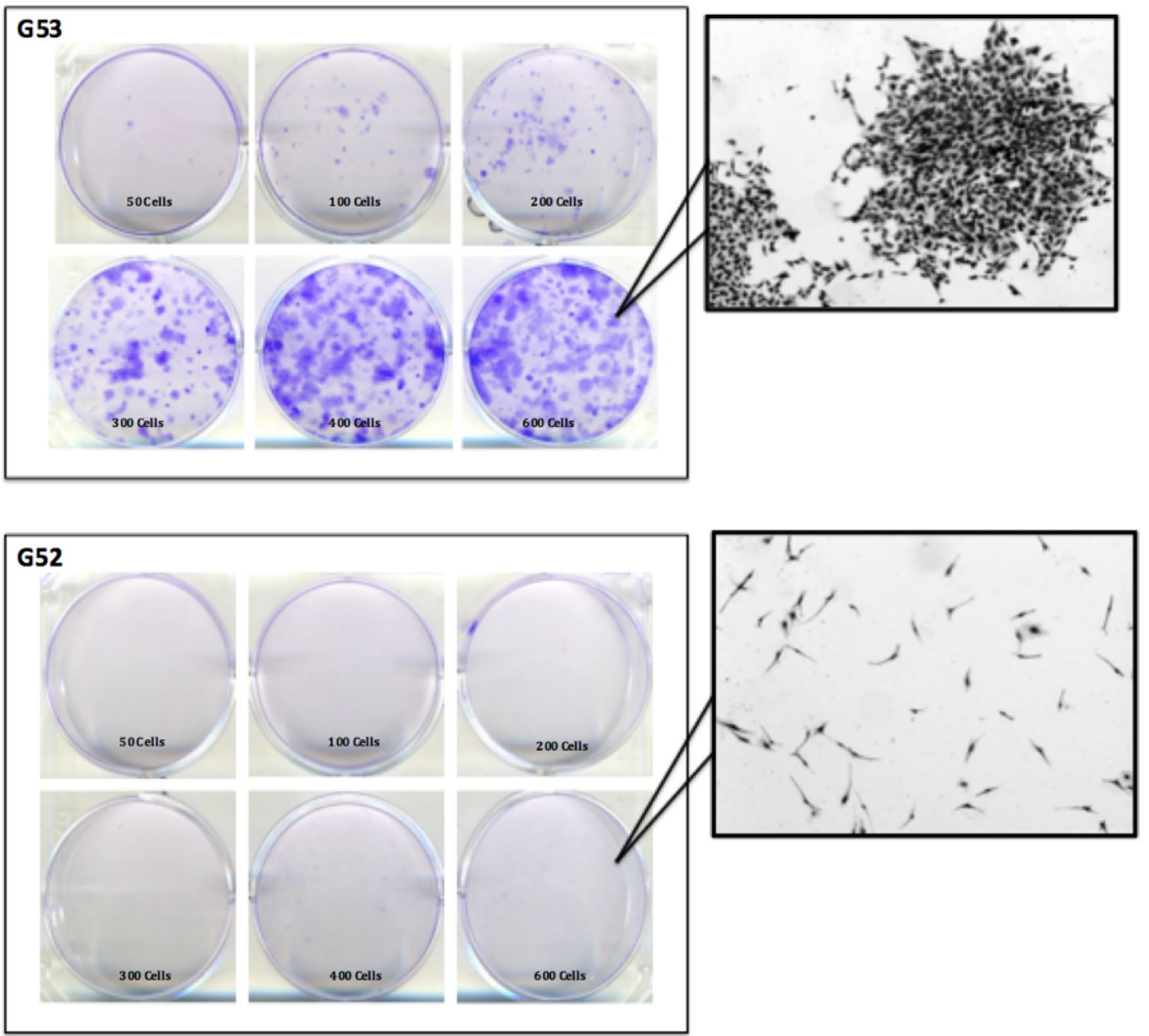

B

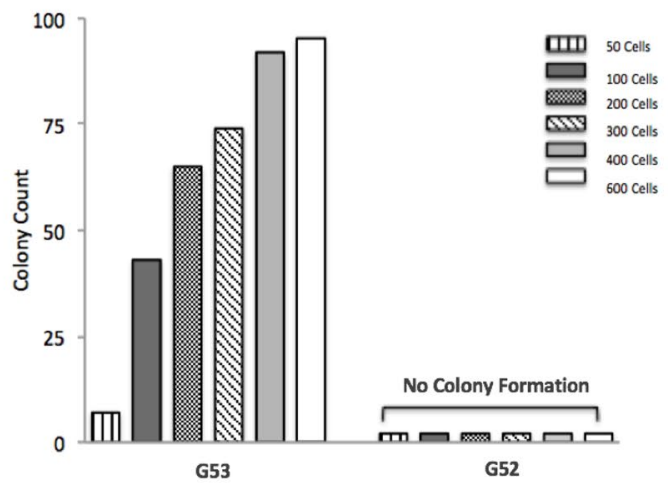

Fig. 4 Colony formation escalation in a p53 mutant cell line. a G53 colony formation (crystal violet stain) increases across all cell seeding densities (50 to 600 cells) over 14 days compared to G52 (inset magnification $\times 20$ ). b Colonies were quantified using a stereomi- croscope and colony counting pen. G53 colony counts consistently increase over time with increasing cell density, whereas G52 demonstrates no change in formation 
A

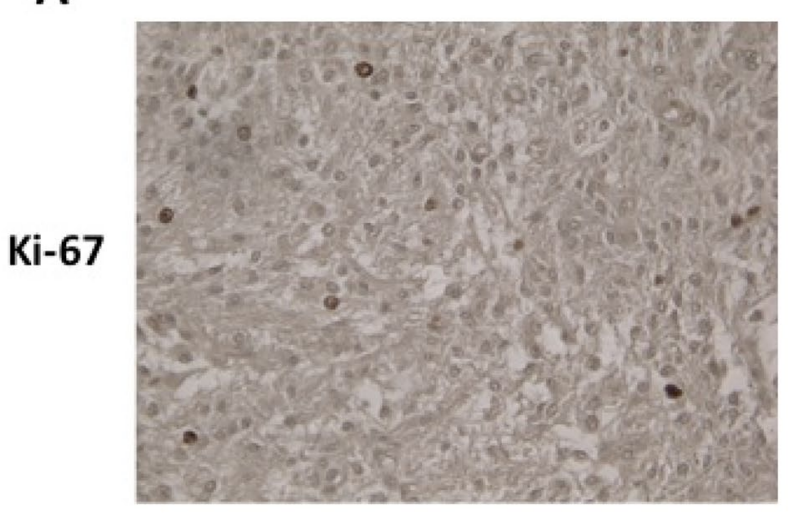

\section{G52}

B

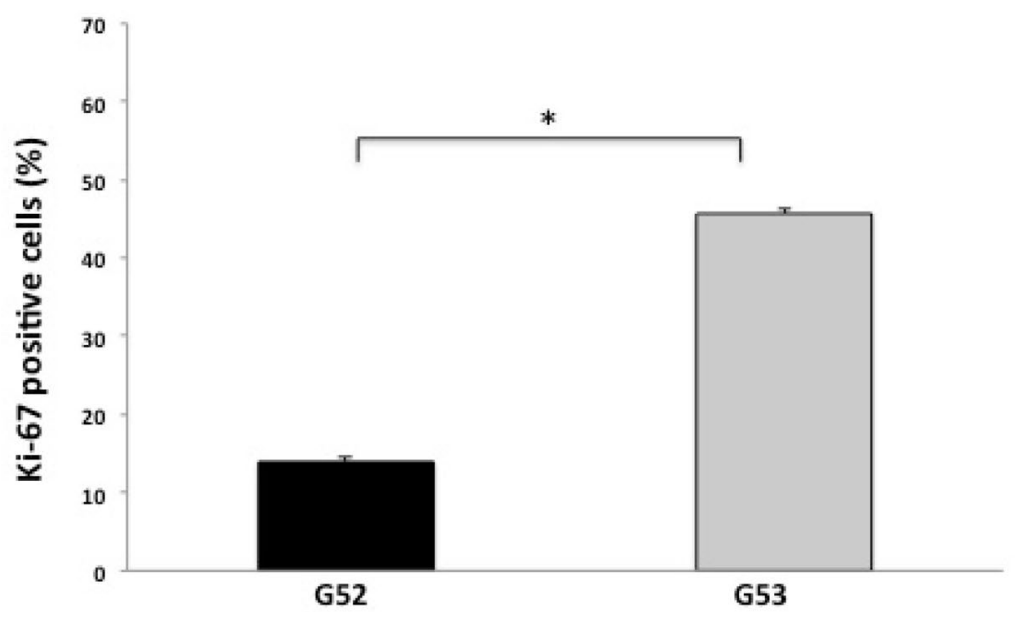

Fig. 5 Higher cell proliferation in p53 mutant. a Labelling index for Ki-67 in G52 and G53 ( $\times 40$ magnification). b LI was measured as the percentage of positive cells per 1000 cells. $* p<0.05$

of a G52 tumour cell that accumulated this aberration during tumorigenesis of G53. The former is the most likely event as p53 mutant cancer cells have an increased ability to migrate to distant sites and metastasise [11]. Either scenario agrees with previous findings that suggest TP53 is not a founder event but rather occurs during the later stages in primary GBM and early stages of secondary GBM tumour development $[25,26]$. When p53 mutations do occur, they result not only in impaired tumour suppressor function, but mounting evidence has demonstrated that certain missense mutations give rise to gain-of-function (GOF) phenotypes.

Numerous studies have demonstrated the contributions of p53 GOF phenotypes to malignancy through mechanisms involving proliferation, migration, invasion, metastasis, drug resistance, colony formation, genomic instability and cancer cell survival $[11,12,27,28]$. In vivo studies have repeatedly displayed an increase in incidence of metastatic neoplasms following the introduction of p53 $\mathrm{R} 270 \mathrm{H}$ [24, 29-31]. We investigated both tumours in vitro to determine if the G53 p53 R273H mutation adopted GOF characteristics and contributed to its aggressive development. The assays demonstrated pronounced increases in proliferation, migration and invasion of G53 compared to G52, strongly indicating that GOF phenotypes were acquired. These results were further validated through a marked increase in colony formation of G53, suggesting the p53 mutation supports cellular growth, results coinciding with previous studies $[32,33]$. Because p53 mutations inhibit DNA binding, it is believed that the initial mechanisms of GOF mutants are mediated mainly through protein-protein interactions with transcription factors such as p63 and p73, or chromatin complexes [34-37]. An alternative mechanism proposes that p53 mutants act as transcription factors with the ability to influence promoters in order to activate target genes [11]. However, the effects of p53 mutants cannot be accurately predicted and the aforementioned mechanisms are likely a combination of differing pathways. The extensive literature combined with our in vitro assays provide likely rationale that TP53 was mutated during the later development of G52. The p53 
mutant subpopulation then migrated from the left thalamus to the left temporal region where metastasis of G53 was established. The mutation provided the tumour with GOF characteristics resulting in a more aggressive tumour with greater oncogenic potential than G52. This particular case emphasises the need for genetic investigation to understand the precise disease progression of $\mathrm{mGBM}$ in order to create highly targeted therapies.

\section{Conclusion}

As the most lethal brain cancer, GBM is characterised by severe genetic instability and a diffusely infiltrative nature. In this MF GBM patient, the secondary tumour acquired a p53 R273H missense mutation, thus establishing a mutational signature with evolutionary advantageous GOF phenotypes. These phenotypes likely contributed to the invasive progression and malignancy of the G53 tumour. The apparent differences in development between the tumours highlights the importance of genomic profiling, particularly in mGBM cases. Understanding each tumour individually provides further insight into the disease and may enhance personalised medicine modalities through molecularly targeted therapy.

Funding This study has been supported by the Cure for Brain Cancer Foundation.

\section{Compliance with ethical standards}

Conflict of interest The authors have declared no conflict of interest.

Ethical approval The patient was enrolled in a Human Research Ethics Committee (HREC)-approved human study at the South Eastern Sydney Local Health District-Northern Sector (Sydney, Australia; HREC No: 2008-094) and consented to publication of photos and their clinical data.

Open Access This article is licensed under a Creative Commons Attribution 4.0 International License, which permits use, sharing, adaptation, distribution and reproduction in any medium or format, as long as you give appropriate credit to the original author(s) and the source, provide a link to the Creative Commons licence, and indicate if changes were made. The images or other third party material in this article are included in the article's Creative Commons licence, unless indicated otherwise in a credit line to the material. If material is not included in the article's Creative Commons licence and your intended use is not permitted by statutory regulation or exceeds the permitted use, you will need to obtain permission directly from the copyright holder. To view a copy of this licence, visit http://creativecommons.org/licenses/by/4.0/.

\section{References}

1. Stupp R et al (2005) Radiotherapy plus concomitant and adjuvant temozolomide for glioblastoma. N Engl J Med 352(10):987-996

2. Lasocki A et al (2016) Multifocal and multicentric glioblastoma: improved characterisation with FLAIR imaging and prognostic implications. J Clin Neurosci 31:92-98

3. Paulsson AK et al (2014) Comparison of clinical outcomes and genomic characteristics of single focus and multifocal glioblastoma. J Neurooncol 119(2):429-435

4. Thomas RP et al (2013) The incidence and significance of multiple lesions in glioblastoma. J Neurooncol 112(1):91-97

5. Hassaneen W et al (2011) Multiple craniotomies in the management of multifocal and multicentric glioblastoma. Clinical article. J Neurosurg 114(3):576-584

6. Batzdorf U, Malamud N (1963) The problem of multicentric gliomas. J Neurosurg 20:122-136

7. Akimoto J et al (2014) Case of radiologically multicentric but genetically identical multiple glioblastomas. Brain Tumor Pathol 31(2):113-117

8. Olivier M, Hollstein M, Hainaut P (2010) TP53 mutations in human cancers: origins, consequences, and clinical use. Cold Spring Harb Perspect Biol 2(1):a001008

9. Robles AI, Harris CC (2010) Clinical outcomes and correlates of TP53 mutations and cancer. Cold Spring Harb Perspect Biol 2(3): $\mathrm{a} 001016$

10. Vogelstein B, Lane D, Levine AJ (2000) Surfing the p53 network. Nature 408(6810):307-310

11. Oren M, Rotter V (2010) Mutant p53 gain-of-function in cancer. Cold Spring Harb Perspect Biol 2(2):a001107

12. Adorno $\mathrm{M}$ et al (2009) A Mutant-p53/Smad complex opposes p63 to empower TGFbeta-induced metastasis. Cell 137(1):87-98

13. Muller PA, Vousden KH, Norman JC (2011) p53 and its mutants in tumor cell migration and invasion. J Cell Biol 192(2):209-218

14. Yue $X$ et al (2017) Mutant p53 in cancer: accumulation, gain-offunction, and therapy. J Mol Biol 429(11):1595-1606

15. Gatalica $Z$ et al (2014) Programmed cell death 1 (PD-1) and its ligand (PD-L1) in common cancers and their correlation with molecular cancer type. Cancer Epidemiol Biomarkers Prev 23(12):2965-2970

16. Millis SZ et al (2015) Molecular profiling of infiltrating urothelial carcinoma of bladder and nonbladder origin. Clin Genitourin Cancer 13(1):e37-49

17. Schroeder B et al (2016) Genetic investigation of multicentric glioblastoma multiforme: case report. J Neurosurg 124(5):1353-1358

18. James CD et al (1988) Clonal genomic alterations in glioma malignancy stages. Cancer Res 48(19):5546-5551

19. Lathia JD et al (2015) Cancer stem cells in glioblastoma. Genes Dev 29(12):1203-1217

20. Sundar SJ et al (2014) The role of cancer stem cells in glioblastoma. Neurosurg Focus 37(6):E6

21. Wang $X$ et al (2014) Gain of function of mutant TP53 in glioblastoma: prognosis and response to temozolomide. Ann Surg Oncol 21(4):1337-1344

22. Petitjean A et al (2007) TP53 mutations in human cancers: functional selection and impact on cancer prognosis and outcomes. Oncogene 26(15):2157-2165

23. Baugh EH et al (2018) Why are there hotspot mutations in the TP53 gene in human cancers? Cell Death Differ 25(1):154-160

24. Muller PA, Vousden KH (2014) Mutant p53 in cancer: new functions and therapeutic opportunities. Cancer Cell 25(3):304-317

25. Abou-El-Ardat K et al (2017) Comprehensive molecular characterization of multifocal glioblastoma proves its monoclonal origin 
and reveals novel insights into clonal evolution and heterogeneity of glioblastomas. Neuro Oncol 19(4):546-557

26. Zhang $\mathrm{Y}$ et al (2018) The p53 pathway in glioblastoma. Cancers (Basel) 10(9):297.

27. Schulz-Heddergott R, UM Moll (2018) Gain-of-function (GOF) mutant p53 as actionable therapeutic target. Cancers (Basel) 10(6): 188

28. Wang SP et al (2009) p53 controls cancer cell invasion by inducing the MDM2-mediated degradation of Slug. Nat Cell Biol 11(6):694-704

29. Olive KP et al (2004) Mutant p53 gain of function in two mouse models of Li-Fraumeni syndrome. Cell 119(6):847-860

30. Heinlein $\mathrm{C}$ et al (2008) Mutant p53(R270H) gain of function phenotype in a mouse model for oncogene-induced mammary carcinogenesis. Int J Cancer 122(8):1701-1709

31. Lang GA et al (2004) Gain of function of a p53 hot spot mutation in a mouse model of Li-Fraumeni syndrome. Cell 119(6):861-872

32. Cho Y et al (1994) Crystal structure of a p53 tumor suppressorDNA complex: understanding tumorigenic mutations. Science 265(5170):346-355
33. Vikhanskaya F et al (2007) Cancer-derived p53 mutants suppress p53-target gene expression-potential mechanism for gain of function of mutant p53. Nucleic Acids Res 35(6):2093-2104

34. Zalcenstein A et al (2006) Repression of the MSP/MST-1 gene contributes to the antiapoptotic gain of function of mutant $\mathrm{p} 53$. Oncogene 25(3):359-369

35. Di Como CJ, Gaiddon C, Prives C (1999) p73 function is inhibited by tumor-derived p53 mutants in mammalian cells. Mol Cell Biol 19(2):1438-1449

36. Li Y, Prives C (2007) Are interactions with p63 and p73 involved in mutant p53 gain of oncogenic function? Oncogene 26(15):2220-2225

37. Strano $\mathrm{S}$ et al (2000) Physical and functional interaction between p53 mutants and different isoforms of p73. J Biol Chem 275(38):29503-29512

Publisher's Note Springer Nature remains neutral with regard to jurisdictional claims in published maps and institutional affiliations. 